\title{
THE BUSY PERIOD OF AN M/G/1 QUEUE WITH CUSTOMER IMPATIENCE
}

\author{
ONNO BOXMA, * EURANDOM and Eindhoven University of Technology \\ DAVID PERRY, ${ }^{* *}$ University of Haifa \\ WOLFGANG STADJE, ${ }^{* * *}$ University of Osnabrück \\ SHELLEY ZACKS, ${ }^{* * * *}$ Binghamton University
}

\begin{abstract}
We consider an M/G/1 queue in which an arriving customer does not enter the system whenever its virtual waiting time, i.e. the amount of work seen upon arrival, is larger than a certain random patience time. We determine the busy period distribution for various choices of the patience time distribution. The main cases under consideration are exponential patience and a discrete patience distribution.
\end{abstract}

Keywords: Busy period; M/G/1 queue; impatience; virtual waiting time;

2000 Mathematics Subject Classification: Primary 60K30

Secondary 60J27; 60J75; 60F03

\section{Introduction}

Impatience is a very natural and important concept in queueing models. There is a wide range of situations in which customers may become impatient when they do not receive service fast enough. We may think of customers at call centers or of customers representing perishable goods, such as blood samples which wait to be tested and become obsolete after a certain due date.

Most of the attention in the literature on queueing models with impatience has focused on queue length and waiting time distributions, with relatively little attention given to the busy period distribution. This important performance measure has been studied by Subba Rao [15] for the $\mathrm{M} / \mathrm{G} / 1+\mathrm{M}$ model, where the notation ' $+\mathrm{M}$ ' indicates exponential patience, and in [8] for the M/G/1 model with restricted accessibility: a customer is fully (or partially) rejected if the workload at his arrival is below a certain fixed threshold. See [6] for the M/M/1+D case, and [7] for several variants of the $M / M / 1+D$ and $M / M / 1+M$ cases.

A pioneering paper on queueing models with impatience is that of Barrer [3], who studied the $\mathrm{M} / \mathrm{M} / \mathrm{s}+\mathrm{D}$ model for the case where impatience refers to the waiting time and the $\mathrm{M} / \mathrm{M} / 1+\mathrm{D}$ model for the case where impatience refers to the sojourn time. In [1] and [2] necessary and sufficient conditions for the existence of the virtual waiting time distribution in the $\mathrm{G} / \mathrm{G} / 1+\mathrm{G}$ queue

Received 11 May 2009; revision received 24 November 2009.

* Postal address: Department of Mathematics and Computer Science, Eindhoven University of Technology, HG 9.14, PO Box 513, 5600 MB Eindhoven, The Netherlands. Email address: boxma@win.tue.nl

** Postal address: Department of Statistics, University of Haifa, Haifa 31909, Israel.

Email address: dperry@haifa.ac.il

*** Postal address: Department of Mathematics and Computer Science, University of Osnabrück, 49069 Osnabrück, Germany. Email address: wolfgang@mathematik.uni-osnabrueck.de

**** Postal address: Department of Mathematical Sciences, Binghamton University, Binghamton, NY 13902-6000, USA. Email address: shelly@math.binghamton.edu 
were obtained. The latter distribution is subsequently obtained for $\mathrm{M} / \mathrm{G} / 1+\mathrm{M}$ and $\mathrm{M} / \mathrm{G} / 1+\mathrm{E}_{k}$ models. Finch [5] derived the waiting time distribution in the G/M/1+D queue. Stanford [12] related the waiting time distribution of the (successful) customers and the workload seen by an arbitrary arrival in the $\mathrm{G} / \mathrm{G} / 1+\mathrm{G}$ queue. See[13] for a brief literature review.

In the present paper we focus on the busy period distribution for a single-server queue with impatience. We consider the $\mathrm{M} / \mathrm{G} / 1+\mathrm{G}$ model, in which the patience refers to the waiting (not sojourn) time of the arriving customer. We first derive an integral equation for the distribution of the busy period length, conditional on the initial workload in the system being $v$. We are able to solve this equation in the case of exponential patience for a large class of service time distributions. We thus obtain the Laplace-Stieltjes transform (LST) of the distribution of the length of a busy period that starts with some workload $v$. Integration with respect to the service time distribution gives the transform of the unconditional busy period length. In the case of a discrete patience distribution, we follow another approach which is based on transform methods, the Wald martingale, and stopping times. Again the LST of the busy period distribution is obtained.

The paper is organized as follows. Section 2 contains a model description, and the derivation of the integral equation for the distribution of the busy period length, conditional on the initial workload in the system being $v$. In Section 3 we exploit this integral equation to obtain the busy period distribution for the case of exponential patience, the service time being either hyperexponential or Erlang distributed. Sections 4, 5, and 6 are devoted to the case of a discrete patience distribution. Section 4 contains the preparations. In Section 5 we consider exponential service times, while in Section 6 we consider generally distributed service times.

\section{An integral equation: model description}

Let $\{V(t), t \geq 0\}$ denote the virtual waiting time (the load) of an M/G/1 queue with arrival intensity $\lambda$. The $n$th customer arrives with a vector of two random variables $\left(X_{n}, U_{n}\right)$, where $X_{n}$ is the length of service required and $U_{n}$ is the patience time. We assume that $\left(X_{1}, X_{2}, \ldots\right)$ and $\left(U_{1}, U_{2}, \ldots\right)$ are two independent sequences of independent and identically distributed (i.i.d.) random variables. The common distribution functions of the $X_{i}$ and $U_{i}$ are denoted by $F$ and $G$, respectively; for simplicity, we assume that they have densities $f$ and $g$, respectively. Let $V(t)$ be the virtual waiting process. If the $n$th customer arrives at time $t$, he sees the workload $V(t-)$ in front of him and joins the queue if and only if $V(t-) \leq U_{n}$; in this case $V(t)=V(t-)+X_{n}$, while, if $V(t-)>U_{n}$, we have $V(t)=V(t-)$.

We are interested in the distribution of the length, $B$, of the busy period in this M/G/1 queue with customer impatience. First we note that $\mathrm{P}\{B<\infty\}=1$ if $\mathrm{E}\left(X_{1}\right)<\infty$. To see this, consider the workload $W_{n}$ just before the arrival of the $n$th customer. This embedded sequence is Markovian and satisfies the recursion

$$
W_{n+1}=\left(W_{n}+X_{n} \mathbf{1}_{\left\{U_{n}>W_{n}\right\}}-Y_{n}\right)^{+},
$$

where $Y_{n}$ denotes the $(\exp (\lambda)$-distributed) time between the arrivals of the $n$th and the $(n+1)$ th customer, and $\mathbf{1}_{A}$ is the indicator function of $A$. Also $(a)^{+}=\max (0, a)$. We have, by dominated convergence,

$$
\mathrm{E}\left(W_{n+1}-W_{n} \mid W_{n}=w\right)=\mathrm{E}\left(\max \left[X_{1} \mathbf{1}_{\left\{U_{1}>w\right\}}-Y_{1},-w\right]\right) \rightarrow-\frac{1}{\lambda} \quad \text { as } w \rightarrow \infty .
$$

Hence, there is a $w_{0}>0$ such that $\mathrm{E}\left(W_{n+1}-W_{n} \mid W_{n}=w\right) \leq-1 / 2 \lambda$ for all $w \geq w_{0}$. It follows that sequence $W_{n}$ almost surely enters and leaves the interval $\left[0, w_{0}\right]$ infinitely often, 
and at any of these visits there is a positive probability that the system will become idle before the workload exceeds $w_{0}$ again. Therefore, $\mathrm{P}\{B<\infty\}=1$.

We now derive an integral equation for the distribution of the busy period duration $B$, initiated by some workload $v$. Let

$$
P(t, v)=\mathrm{P}\{B>t \mid V(0)=v\}, \quad t \geq 0, v>0 .
$$

We start from the following renewal equation (cf., e.g. [9]):

$$
\begin{aligned}
P(t, v)= & \mathrm{e}^{-\lambda t} \mathbf{1}_{\{v>t\}} \\
& +\lambda \int_{0}^{\min (t, v)} \mathrm{e}^{-\lambda s} \bar{G}(v-s) \int_{0}^{\infty} P(t-s, v-s+x) f(x) \mathrm{d} x \mathrm{~d} s \\
& +\lambda \int_{0}^{\min (t, v)} \mathrm{e}^{-\lambda s} G(v-s) P(t-s, v-s) \mathrm{d} s,
\end{aligned}
$$

where $\bar{G}=1-G$. Using Banach's fixed point theorem, we can uniformly approximate the function $(t, v) \mapsto P(t, v)$ on $[0, T] \times(0, \infty)$ for arbitrary $T>0$. Let $\mathscr{B}_{T}$ be the Banach space of all measurable and bounded real-valued functions on $[0, T] \times(0, \infty)$, endowed with the supremum norm $\|\cdot\|_{\infty}$, and define an operator $\mathcal{A}: \mathscr{B}_{T} \rightarrow \mathcal{B}_{T}$ as follows. Define $(\mathcal{A} h)(t, v)$ to be the right-hand side of $(2.1)$, replacing $P(\cdot, \cdot)$ by $h(\cdot, \cdot)$ for $h \in \mathscr{B}_{T}$ and $(t, v) \in[0, T] \times$ $(0, \infty)$. A simple calculation shows that, for any $h, \bar{h} \in \mathcal{B}_{T}$,

$$
\|\mathcal{A} h-\mathcal{A} \bar{h}\|_{\infty} \leq\left(1-\mathrm{e}^{-\lambda T}\right)\|h-\bar{h}\|_{\infty} .
$$

Thus, $\mathcal{A}$ is a contraction on $\mathcal{B}_{T}$, whose unique fixed point is the function $P(t, v),(t, v) \in$ $[0, T] \times(0, \infty)$, and, for every initial function $h_{0} \in \mathscr{B}_{T}$, the sequence defined recursively by $h_{n+1}=\mathcal{A} h_{n}, n \geq 0$, converges uniformly to the fixed point at a geometric rate.

We can obtain more explicit results in many important special cases by transforming (2.1) into an integro-differential equation as follows. Introducing the Laplace transform $P^{*}(\theta, v)=$ $\int_{0}^{\infty} \mathrm{e}^{-\theta t} P(t, v) \mathrm{d} t$, it follows after some manipulations that

$$
\begin{aligned}
P^{*}(\theta, v)= & \frac{1}{\lambda+\theta}\left(1-\mathrm{e}^{-(\lambda+\theta) v}\right) \\
& +\lambda \mathrm{e}^{-(\lambda+\theta) v}\left[\int_{0}^{v} \bar{G}(z) \mathrm{e}^{(\lambda+\theta) z} \int_{z}^{\infty} f(y-z) P^{*}(\theta, y) \mathrm{d} y \mathrm{~d} z\right. \\
& \left.+\int_{0}^{v} G(z) \mathrm{e}^{(\lambda+\theta) z} P^{*}(\theta, z) \mathrm{d} z\right]
\end{aligned}
$$

Differentiation with respect to $v$ yields an integro-differential equation for $P^{*}(\theta, \cdot)$ :

$$
\begin{aligned}
\frac{\mathrm{d}}{\mathrm{d} v} P^{*}(\theta, v)= & \mathrm{e}^{-(\lambda+\theta) v}-(\lambda+\theta)\left[P^{*}(\theta, v)-\frac{1}{\lambda+\theta}\left(1-\mathrm{e}^{-(\lambda+\theta) v}\right)\right] \\
& +\lambda \mathrm{e}^{-(\lambda+\theta) v}\left[\bar{G}(v) \mathrm{e}^{(\lambda+\theta) v} \int_{v}^{\infty} f(y-v) P^{*}(\theta, y) \mathrm{d} y+G(v) \mathrm{e}^{(\lambda+\theta) v} P^{*}(\theta, v)\right] \\
= & -(\lambda+\theta) P^{*}(\theta, v)+1+\lambda \bar{G}(v) \int_{v}^{\infty} f(y-v) P^{*}(\theta, y) \mathrm{d} y+\lambda G(v) P^{*}(\theta, v) .
\end{aligned}
$$




\section{Exponential patience}

In this section we exploit the integral equation (2.3) to derive the busy period distribution in the case of exponential patience. We shall consider the following service time distributions: hyperexponential (case (a)), Erlang (case (b)), and finally exponential (case (c)). From the analysis of cases (a) and (b), it is not difficult to figure out how more general combinations of hyperexponential and Erlang service time distributions can be handled.

If $G(v)=1-\mathrm{e}^{-\xi v}, v>0$, then (2.3) reduces to

$$
\frac{\mathrm{d}}{\mathrm{d} v} P^{*}(\theta, v)=-\left(\lambda \mathrm{e}^{-\xi v}+\theta\right) P^{*}(\theta, v)+1+\lambda \mathrm{e}^{-\xi v} \int_{v}^{\infty} f(y-v) P^{*}(\theta, y) \mathrm{d} y, \quad v>0 .
$$

Introduce the double Laplace transform

$$
\pi(\theta, \alpha)=\int_{0}^{\infty} \mathrm{e}^{-\alpha v} P^{*}(\theta, v) \mathrm{d} v
$$

Since $P^{*}(\theta, 0)=0$, we obtain, from (3.1),

$\alpha \pi(\theta, \alpha)=-\theta \pi(\theta, \alpha)-\lambda \pi(\theta, \alpha+\xi)+\frac{1}{\alpha}+\lambda \int_{v=0}^{\infty} \mathrm{e}^{-(\alpha+\xi) v} \int_{y=v}^{\infty} f(y-v) P^{*}(\theta, y) \mathrm{d} y \mathrm{~d} v$.

We try to tackle this integral equation via the following observation. The inversion formula for Laplace transforms (cf. [16]) reads, for some positive $a$,

$$
P^{*}(\theta, y)=\frac{1}{2 \pi \mathrm{i}} \int_{a-\mathrm{i} \infty}^{a+\mathrm{i} \infty} \mathrm{e}^{y s} \pi(\theta, s) \mathrm{d} s .
$$

Substitution into (3.3) gives

$$
\begin{aligned}
(\alpha+\theta) \pi(\theta, \alpha)= & -\lambda \pi(\theta, \alpha+\xi)+\frac{1}{\alpha} \\
& +\frac{\lambda}{2 \pi \mathrm{i}} \int_{a-\mathrm{i} \infty}^{a+\mathrm{i} \infty} \pi(\theta, s) \int_{v=0}^{\infty} \mathrm{e}^{-(\alpha+\xi) v} \int_{y=v}^{\infty} f(y-v) \mathrm{e}^{y s} \mathrm{~d} y \mathrm{~d} v \mathrm{~d} s \\
= & -\lambda \pi(\theta, \alpha+\xi)+\frac{1}{\alpha}+\frac{\lambda}{2 \pi \mathrm{i}} \int_{a-\mathrm{i} \infty}^{a+\mathrm{i} \infty} \pi(\theta, s) \frac{\phi(-s)}{\alpha+\xi-s} \mathrm{~d} s,
\end{aligned}
$$

where $\phi(\cdot)$ denotes the Laplace transform of the service time density. Here $\pi(\theta, s)$ and $\phi(-s)$ are both well defined on $\operatorname{Re} s=0$.

Case (a): $M / H_{N} / l+M$. In the case of a hyperexponential service time density,

$$
f(y)=\sum_{i=1}^{N} p_{i} \mu_{i} \mathrm{e}^{-\mu_{i} y}, \quad \text { where } \quad p_{i}>0, i=1, \ldots, N, \text { and } \sum_{i=1}^{N} p_{i}=1,
$$

(3.4) reduces to

$$
(\alpha+\theta) \pi(\theta, \alpha)=-\lambda \pi(\theta, \alpha+\xi)+\frac{1}{\alpha}+\frac{\lambda}{2 \pi \mathrm{i}} \int_{a-\mathrm{i} \infty}^{a+\mathrm{i} \infty} \frac{\pi(\theta, s)}{\alpha+\xi-s} \sum_{i=1}^{N} p_{i} \frac{\mu_{i}}{\mu_{i}-s} \mathrm{~d} s .
$$


The integrand has $N+1$ poles $s_{0}=\alpha+\xi, s_{i}=\mu_{i}, i=1, \ldots, N$, all in the right-hand half-plane. So replace the integral from $a-\mathrm{i} \infty$ to $a+\mathrm{i} \infty$ by the integral over the closed contour consisting of a line through $a$, parallel to the imaginary axis, and the semi-circle in the right-hand half-plane with the origin at $a$ and radius $R$, and let $R \rightarrow \infty$. Use Cauchy's theorem to conclude that, following the contour in the counterclockwise direction, the integral equals minus the sum of the residues. The contribution of the integral along the semi-circle disappears for $R \rightarrow \infty$. Hence, (3.5) reduces to

$$
\begin{aligned}
(\alpha+\theta) \pi(\theta, \alpha)= & -\lambda \pi(\theta, \alpha+\xi)+\frac{1}{\alpha}-\lambda \sum_{i=1}^{N} p_{i} \frac{\mu_{i}}{\alpha+\xi-\mu_{i}} \pi(\theta, \alpha+\xi) \\
& +\lambda \sum_{i=1}^{N} p_{i} \frac{\mu_{i}}{\alpha+\xi-\mu_{i}} \pi\left(\theta, \mu_{i}\right)
\end{aligned}
$$

or

$$
\begin{aligned}
\pi(\theta, \alpha)= & \frac{1}{\alpha(\alpha+\theta)}-\frac{\lambda}{\alpha+\theta}\left(1+\sum_{i=1}^{N} p_{i} \frac{\mu_{i}}{\alpha+\xi-\mu_{i}}\right) \pi(\theta, \alpha+\xi) \\
& +\frac{\lambda}{\alpha+\theta} \sum_{i=1}^{N} p_{i} \frac{\mu_{i}}{\alpha+\xi-\mu_{i}} \pi\left(\theta, \mu_{i}\right)
\end{aligned}
$$

This equation has the form

$$
\pi(\theta, \alpha)=A_{1}(\theta, \alpha)+A_{2}(\theta, \alpha) \pi(\theta, \alpha+\xi),
$$

where

$$
\begin{aligned}
& A_{1}(\theta, \alpha)=\frac{1}{\alpha(\alpha+\theta)}+\frac{\lambda}{\alpha+\theta} \sum_{i=1}^{N} p_{i} \frac{\mu_{i}}{\alpha+\xi-\mu_{i}} \pi\left(\theta, \mu_{i}\right), \\
& A_{2}(\theta, \alpha)=-\frac{\lambda}{\alpha+\theta}\left(1+\sum_{i=1}^{N} p_{i} \frac{\mu_{i}}{\alpha+\xi-\mu_{i}}\right) .
\end{aligned}
$$

Note that, for any fixed $\theta>0$,

$$
A_{1}(\theta, \alpha)=O\left(\frac{1}{\alpha^{2}}\right) \quad \text { and } \quad A_{2}(\theta, \alpha)=O\left(\frac{1}{\alpha}\right) \quad \text { as } \alpha \rightarrow \infty .
$$

Upon iteration of (3.6) (replacing $\alpha$ by $\alpha+\xi$ on the left-hand side, etc.) we obtain

$$
\pi(\theta, \alpha)=\sum_{j=0}^{\infty} A_{1}(\theta, \alpha+j \xi) \prod_{i=0}^{j-1} A_{2}(\theta, \alpha+i \xi) .
$$

Note that the $j$ th term in the series in (3.7) is bounded by $\left(C_{1} /(1+j)^{2}\right) \prod_{i=0}^{j-1}\left(C_{2} /(1+i)\right) \leq$ $C_{1} C_{2}^{j} /(j+1)$ ! for certain constants $C_{1}$ and $C_{2}$ depending on $\alpha$ and $\theta$. Therefore, the convergence of this series of products is ensured. The expression contains $N$ unknowns $\pi\left(\theta, \mu_{i}\right)$. They can be found by substituting $\alpha=\mu_{i}$ into (3.7) for $i=1, \ldots, N$, yielding $N$ linear equations for these $N$ unknowns. 
Remark 3.1. Recall the meaning of the double Laplace transform $\pi(\theta, \alpha)$ given in (3.2). Then it is seen that a weighted sum of the $\pi\left(\theta, \mu_{i}\right)$ yields the LST of the distribution of the length of a busy period initiated by a customer arriving in an empty system:

$$
\sum_{i=1}^{N} p_{i} \mu_{i} \pi\left(\theta, \mu_{i}\right)=\int_{t=0}^{\infty} \mathrm{e}^{-\theta t} \mathrm{P}\{B>t\} \mathrm{d} t=\frac{1-\mathrm{E}\left(\mathrm{e}^{-\theta B}\right)}{\theta} .
$$

Case $(b): M / E_{k} / l+M$. The case of an Erlang service time density may be treated in a similar way as the hyperexponential case, starting from (3.4). However, since the present paper is methodologically oriented, aiming to explain various methods to handle the busy period problem, we prefer to show an alternative method, which we could also have applied in case (a). Substituting the Erlang- $k$ density in (3.3) and interchanging integrals, it follows that

$$
\begin{aligned}
\pi(\theta, \alpha)= & -\frac{\lambda}{\alpha+\theta} \pi(\theta, \alpha+\xi)+\frac{1}{\alpha(\alpha+\theta)} \\
& +\frac{\lambda}{\alpha+\theta} \int_{v=0}^{\infty} \mathrm{e}^{-(\alpha+\xi) v} \int_{y=v}^{\infty} \mu^{k} \frac{(y-v)^{k-1}}{(k-1) !} \mathrm{e}^{-\mu(y-v)} P^{*}(\theta, y) \mathrm{d} y \mathrm{~d} v \\
= & -\frac{\lambda}{\alpha+\theta} \pi(\theta, \alpha+\xi)+\frac{1}{\alpha(\alpha+\theta)} \\
& +\left.\frac{\lambda}{\alpha+\theta} \mu^{k} \frac{(-1)^{k-1}}{(k-1) !} \frac{\mathrm{d}^{k-1}}{\mathrm{~d} z^{k-1}}\left[\int_{v=0}^{\infty} \mathrm{e}^{-(\alpha+\xi) v} \int_{y=v}^{\infty} \mathrm{e}^{-z(y-v)} P^{*}(\theta, y) \mathrm{d} y \mathrm{~d} v\right]\right|_{z=\mu} \\
= & -\frac{\lambda}{\alpha+\theta} \pi(\theta, \alpha+\xi)+\frac{1}{\alpha(\alpha+\theta)} \\
& +\left.\frac{\lambda}{\alpha+\theta} \mu^{k} \frac{(-1)^{k-1}}{(k-1) !} \frac{\mathrm{d}^{k-1}}{\mathrm{~d} z^{k-1}}\left[\frac{1}{\alpha+\xi-z}(\pi(\theta, z)-\pi(\theta, \alpha+\xi))\right]\right|_{z=\mu} \\
= & -\frac{\lambda}{\alpha+\theta} \pi(\theta, \alpha+\xi)+\frac{1}{\alpha(\alpha+\theta)} \\
& +\frac{\lambda}{\alpha+\theta} \frac{\mu^{k}}{(\mu-\xi-\alpha)^{k}} \pi(\theta, \alpha+\xi)+\left.\frac{\lambda}{\alpha+\theta} \mu^{k} \frac{(-1)^{k-1}}{(k-1) !} \frac{\mathrm{d}^{k-1}}{\mathrm{~d} z^{k-1}} \frac{\pi(\theta, z)}{\alpha+\xi-z}\right|_{z=\mu}
\end{aligned}
$$

Note that, after iterating, the structure of the resulting expression is the same as that of (3.7), except that the $\pi\left(\theta, \mu_{i}\right)$ terms are replaced by $\left.\left(\mathrm{d}^{j} / \mathrm{d} z^{j}\right) \pi(\theta, z)\right|_{z=\mu}$ terms. To determine $\pi(\theta, \mu)$ and those $k-1$ derivatives, we have to differentiate the resulting expression $k-1$ times, substituting $\alpha=\mu$.

Case (c): $M / M / 1+M$. The $\mathrm{M} / \mathrm{M} / 1$ queue with exponential patience forms a special case of both cases (a) and (b). If we do not wish to determine $\pi(\theta, \alpha)$, but are satisfied with the busy period LST, then the birth-and-death approach of [7] works well in the $M / M / 1+M$ case. That approach in particular leads to the following expression for the mean busy period:

$$
\mathrm{E}(B)=\sum_{k=0}^{\infty} \frac{\lambda^{k}}{\mu(\mu+\xi) \cdots(\mu+k \xi)} .
$$

On the other hand, it follows from (3.7) that

$$
\pi(0, \mu)=\sum_{j=0}^{\infty}\left[\frac{1}{(\mu+j \xi)^{2}}+\frac{\lambda \mu}{(j+1) \xi} \frac{\pi(0, \mu)}{\mu+j \xi}\right] \prod_{i=0}^{j-1} A_{2}(0, \mu+i \xi) .
$$


Hence

$$
\begin{aligned}
\mathrm{E}(B)= & \mu \pi(0, \mu) \\
= & \left(\mu \sum_{j=0}^{\infty} \frac{1}{(\mu+j \xi)^{2}} \prod_{i=0}^{j-1} A_{2}(0, \mu+i \xi)\right) \\
& \times\left(1-\sum_{j=0}^{\infty} \frac{\lambda \mu}{(j+1) \xi} \frac{1}{\mu+j \xi} \prod_{i=0}^{j-1} A_{2}(0, \mu+i \xi)\right)^{-1} .
\end{aligned}
$$

Here

$$
A_{2}(0, z)=-\frac{\lambda}{z} \frac{z+\xi}{z+\xi-\mu}
$$

and, hence,

$$
\prod_{i=0}^{j-1} A_{2}(0, \mu+i \xi)=\frac{(-\lambda / \xi)^{j}}{j !} \frac{\mu+j \xi}{\mu} .
$$

Substitution into (3.9) gives a second explicit series representation of $\mathrm{E}(B)$ :

$$
\mathrm{E}(B)=\mathrm{e}^{\lambda / \xi} \sum_{j=0}^{\infty} \frac{(-\lambda / \xi)^{j}}{j !} \frac{1}{\mu+j \xi} .
$$

The expressions in (3.8) and (3.10) agree. It can be shown, by complete induction, that

$$
\frac{\lambda^{k}}{\mu(\mu+\xi) \cdots(\mu+k \xi)}=\frac{(\lambda / \xi)^{k}}{k !} \sum_{j=0}^{k} \frac{\left(\begin{array}{l}
k \\
j
\end{array}\right)(-1)^{j}}{\mu+j \xi} .
$$

Summing from $k=0$ to $\infty$ and interchanging the sums on the right-hand side indeed confirms the equivalence of (3.8) and (3.10).

Remark 3.2. Our analysis of the busy period in the $M / G / 1+M$ queue differs in several respects from the analysis of Subba Rao [15]. Subba Rao considered the M/G/1+M queue with the additional feature of balking with a constant probability. If, upon arrival, a customer finds $n$ customers present, with $n \geq 1$, then it balks (i.e. leaves immediately) with a fixed probability $1-\beta$. If it finds an empty system, it always joins the system. Here $\beta=1$ obviously removes the balking feature from the model.

Using supplementary variable techniques and complex function theory, Subba Rao [15] derived an expression for the double transform of the joint distribution of the number of customers served during a busy period and the length of that period, given that it starts with $i+1$ customers in the system. This expression is in the form of a quotient of double sums. Our analysis does not consider the number of customers served, and starts from a given amount of work at the beginning of the busy period (instead of a given number of customers). In [14], Subba Rao considered the combined effects of balking and customer impatience (also called reneging) for the case where the balking probability is $b_{n}$ if the arriving customer meets $n$ customers.

Remark 3.3. Equations (2.2) and (2.3) also hold in the $\mathrm{M} / \mathrm{G} / 1+\mathrm{G}$ case, i.e. when $F$ or $G$ or both do not have Lebesgue densities. We only have to replace the term $\int_{z}^{\infty} f(y-z) P^{*}(\theta, y) \mathrm{d} y$ by $\int_{0}^{\infty} P^{*}(\theta, u+z) \mathrm{d} F(u)$. In particular, for the M/G/1+D system with deterministic impatience 
times, the above techniques can also be used for special choices of the service time distribution. In the case of several patience levels, i.e. a discrete patience distribution, this technique becomes very involved. In the next three sections we develop another approach which seems better suited to deal with deterministic patience and can even handle models with several patience levels.

\section{A discrete patience distribution}

In this section and the next two sections we assume that the patience random variables $U_{1}, U_{2}, \ldots$ have a discrete distribution concentrated on the set $\left\{v_{1}, \ldots, v_{K}\right\}$ with probabilities $p_{i}=\mathrm{P}\left\{U=v_{i}\right\}, i=1, \ldots, K, \sum_{i=1}^{K} p_{i}=1$, where $v_{i-1}<v_{i}, i=1, \ldots, K, v_{0} \equiv 0$, and $v_{K}<\infty$. Let $P(u)$ be the corresponding cumulative distribution function (CDF), i.e.

$$
P(u)=\sum_{v_{i} \leq u} p_{i}
$$

Consider the corresponding partition of the positive orthant $\left\{\mathcal{V}_{1}, \mathcal{V}_{2}, \ldots, \mathcal{V}_{K+1}\right\}$, where

$$
\mathcal{V}_{i}=\left\{(t, v): 0 \leq t<\infty, v_{i-1} \leq v<v_{i}\right\}, \quad i=1, \ldots, K,
$$

and

$$
\mathcal{V}_{K+1}=\left\{(t, v): 0 \leq t<\infty, v_{K} \leq v<\infty\right\},
$$

where $v_{0} \equiv 0$. Accordingly, if $V(t) \in \mathcal{V}_{1}$, all customers join the queue. Generally, if $V(t) \in \mathcal{V}_{i}$ $(i=1, \ldots, K+1)$, the probability that a customer will join the queue is $Q_{i-1}=1-P\left(v_{i-1}\right)$. Note that $Q_{0}=1, Q_{i}<Q_{i-1}$ for all $i=1, \ldots, K$, and $Q_{K}=0$. We observe that an arbitrary patience time distribution may be approximated by the above discrete distribution, by choosing $K$ and the probabilities $p_{i}$ such that the first moments of the patience time distribution match.

Customers arrive at the queue to an ordinary Poisson process (OPP) with intensity $\lambda$. Owing to the strong Markov property, customers join the queue according to an OPP with intensity $\lambda_{i}=\lambda Q_{i-1}(i=1, \ldots, K+1)$ during periods in which the $\{V(t)\}$ process is in region $\mathcal{V}_{i}$. Let $\left\{V^{(i)}(t)\right\}$ designate the $V(t)$ process in the region $\mathcal{V}_{i}(i=1, \ldots, K)$.

The busy period, with length $B$, starts with $V^{(i)}(0)=X_{1}$ and terminates as soon as $V^{(1)}(t)=$ 0 . If $V^{(1)}(t)$ crosses from $\mathcal{V}_{1}$ to $\mathcal{V}_{2}$ before hitting the value zero then a new process, $V^{(2)}(t)$ say, starts. This process will either return to $\mathcal{V}_{1}$ before hitting the upper boundary of $\mathcal{V}_{2}$, or will enter $\mathcal{V}_{3}$ first, and so on. Our aim is to derive the LST of $B$. In the next section we start with a recursive construction of this LST for an $\mathrm{M} / \mathrm{M} / 1$ queue. We then generalize the results.

\section{The busy period LST for an $M / M / 1$ queue with discrete patience}

\subsection{Auxiliary results}

The M/M/1 queue is based on the compound Poisson process $Y(t)=\sum_{n=0}^{N(t)} X_{n}$, where $\{N(t), t \geq 0\}$ is an OPP with intensity $\lambda, 0<\lambda<\infty$, and $X_{0} \equiv 0, X_{1}, X_{2}, \ldots$ are i.i.d. random variables having an exponential distribution, $\exp (\mu)$, where $\mathrm{E}\left(X_{1}\right)=1 / \mu$. For constants $0<\beta_{1}, \beta_{2}<\infty$, we define the stopping variables

$$
T_{L}\left(\beta_{1}\right)=\inf \left\{t>0: Y(t)=-\beta_{1}+t\right\}
$$

and

$$
T_{U}\left(\beta_{2}\right)=\inf \left\{t>0: Y(t) \geq \beta_{2}+t\right\} .
$$


In addition, let

$$
T\left(\beta_{1}, \beta_{2}\right)=\min \left\{T_{L}\left(\beta_{1}\right), T_{U}\left(\beta_{2}\right)\right\}
$$

and observe that

$$
\mathrm{P}\left\{T\left(\beta_{1}, \beta_{2}\right)<\infty\right\} \geq \mathrm{P}\left\{T_{L}\left(\beta_{1}\right)<\infty\right\}=1 .
$$

We need formulae for the transforms

$$
\psi_{L}^{*}\left(\omega \mid \beta_{1}, \beta_{2}\right)=\mathrm{E}\left(\mathrm{e}^{-\omega T_{L}\left(\beta_{1}\right)} \mathbf{1}_{\left\{T_{L}\left(\beta_{1}\right)<T_{U}\left(\beta_{2}\right)\right\}}\right)
$$

and

$$
\psi_{U}^{*}\left(\omega \mid \beta_{1}, \beta_{2}\right)=\mathrm{E}\left(\mathrm{e}^{-\omega T_{U}\left(\beta_{2}\right)} \mathbf{1}_{\left\{T_{L}\left(\beta_{1}\right)>T_{U}\left(\beta_{2}\right)\right\}}\right) .
$$

In the $\mathrm{M} / \mathrm{M} / 1$ case, the Wald martingale [10] yields the identity

$$
\mathrm{E}\left(\exp \left\{-\theta Y\left(T\left(\beta_{1}, \beta_{2}\right)\right)+\frac{\lambda T\left(\beta_{1}, \beta_{2}\right) \theta}{\mu+\theta}\right\}\right)=1
$$

for all $\theta>-\mu$. From this fundamental identity we obtain the formulae

$$
\psi_{L}^{*}\left(\omega \mid \beta_{1}, \beta_{2}\right)=\frac{\left(\mu+\theta_{2}(\omega)\right) \mathrm{e}^{-\beta_{2} \theta_{1}(\omega)}-\left(\mu+\theta_{1}(\omega)\right) \mathrm{e}^{-\beta_{2} \theta_{2}(\omega)}}{D\left(\omega \mid \beta_{1}, \beta_{2}\right)}
$$

and

$$
\psi_{U}^{*}\left(\omega \mid \beta_{1}, \beta_{2}\right)=\frac{\mathrm{e}^{\beta_{1} \theta_{2}(\omega)}-\mathrm{e}^{\beta_{1} \theta_{1}(\omega)}}{D\left(\omega \mid \beta_{1}, \beta_{2}\right)}
$$

where

$$
\theta_{1,2}(\omega)=\frac{1}{2}(\lambda-\mu+\omega) \pm \frac{1}{2} \sqrt{(\lambda-\mu+\omega)^{2}+4 \omega \mu}
$$

and

$$
\begin{aligned}
D\left(\omega \mid \beta_{1}, \beta_{2}\right)= & \left(\mu+\theta_{2}(\omega)\right) \exp \left\{-\beta_{2} \theta_{1}(\omega)+\beta_{1} \theta_{2}(\omega)\right\} \\
& -\left(\mu+\theta_{1}(\omega)\right) \exp \left\{-\beta_{2} \theta_{2}(\omega)+\beta_{1} \theta_{1}(\omega)\right\} .
\end{aligned}
$$

\subsection{The busy period LST when $K=1$}

The case in which $K=1$ corresponds to an $\mathrm{M} / \mathrm{M} / 1$ queue with deterministic $\left(v_{1}\right)$ patience; see also [6] for a treatment of this case. In this case, $\lambda_{1}=\lambda$ and $\lambda_{2}=0$. Let $\delta_{1}=v_{1}$, and consider the cases where $X_{1}<v_{1}$ and $X_{1} \geq v_{1}$.

Case 1: $X_{1}<v_{1}$. Since $V^{(1)}(0)=X_{1}<v_{1}$, set $\beta_{1}=X_{1}$ and $\beta_{2}=\delta_{1}-X_{1}$. Substituting these into (5.3) and (5.4) we obtain, after some algebraic manipulations,

$$
\psi_{L}^{*}\left(\omega \mid X, \delta_{1}-X\right)=\zeta_{1}(\omega) \mathrm{e}^{-X \theta_{2}(\omega)}-\zeta_{2}(\omega) \mathrm{e}^{-X \theta_{1}(\omega)},
$$

where

$$
\begin{aligned}
& \zeta_{1}(\omega)=\frac{\left(\mu+\theta_{2}(\omega)\right) \mathrm{e}^{-\delta_{1} \theta_{1}(\omega)}}{\left(\mu+\theta_{2}(\omega)\right) \mathrm{e}^{-\delta_{1} \theta_{1}(\omega)}-\left(\mu+\theta_{1}(\omega)\right) \mathrm{e}^{-\delta_{1} \theta_{2}(\omega)}}, \\
& \zeta_{2}(\omega)=\frac{\left(\mu+\theta_{1}(\omega)\right) \mathrm{e}^{-\delta_{1} \theta_{2}(\omega)}}{\left(\mu+\theta_{2}(\omega)\right) \mathrm{e}^{-\delta_{1} \theta_{1}(\omega)}-\left(\mu+\theta_{1}(\omega)\right) \mathrm{e}^{-\delta_{1} \theta_{2}(\omega)}} .
\end{aligned}
$$

Similarly,

$$
\psi_{U}^{*}\left(\omega \mid X, \delta_{1}-X\right)=\frac{\mathrm{e}^{-X \theta_{1}(\omega)}-\mathrm{e}^{-X \theta_{2}(\omega)}}{\left(\mu+\theta_{2}(\omega)\right) \mathrm{e}^{-\delta_{1} \theta_{1}(\omega)}-\left(\mu+\theta_{1}(\omega)\right) \mathrm{e}^{-\delta_{1} \theta_{2}(\omega)}} .
$$


Whenever $V(t)$ jumps over the boundary $v_{1}$, the overshoot $R$ is independent of $T_{U}\left(\beta_{2}\right)$ and exponentially distributed. The sojourn time of $V(t)$ in $\mathcal{V}_{2}$ is exactly $R$. The LST of $R$ is $\mu /(\mu+\omega)$. The time interval $\left(0, T_{U}\left(\beta_{2}\right)+R\right)$ is called an initial phase. At the end of the initial phase the process $V^{(1)}(t)$ may jump again above $v_{1}$, or may go down to 0 . The times between consecutive returns to $\mathcal{V}_{1}$ are called renewal cycles. If after an initial or a renewal cycle $V^{(1)}(t)$ hits 0 , then the time interval is called a terminal phase. The lengths of renewal cycles are i.i.d. random variables. Thus, the conditional LST of $B$ given $X_{1}$ is, in case 1 ,

$$
\begin{aligned}
M^{(1)}\left(\omega \mid X_{1}, \delta_{1}\right)= & \psi_{L}^{*}\left(\omega \mid X_{1}, \delta_{1}-X_{1}\right) \\
& +\frac{\mu}{\mu+\omega} \psi_{U}^{*}\left(\omega \mid X_{1}, \delta_{1}-X_{1}\right) \frac{\psi_{L}^{*}\left(\omega \mid \delta_{1}, 0\right)}{1-\mu \psi_{U}^{*}\left(\omega \mid \delta_{1}, 0\right) /(\mu+\omega)} .
\end{aligned}
$$

Case 2: $X_{1} \geq v_{1}$. Let $R_{1}=X_{1}-v_{1}$. The initial phase consists only of $R_{1}$. Here $R_{1}$ is independent of the following cycles. Thus, when $X_{1} \geq v_{1}$, the LST is

$$
\tilde{M}^{(1)}\left(\omega \mid \delta_{1}\right)=\frac{\mu}{\mu+\omega} \frac{\psi_{L}^{*}\left(\omega \mid \delta_{1}, 0\right)}{1-\mu \psi_{U}^{*}\left(\omega \mid \delta_{1}, 0\right) /(\mu+\omega)} .
$$

Finally, the LST of $B$, when $K=1$, is

$$
M^{(1)}\left(\omega \mid \delta_{1}\right)=\mu \int_{0}^{\delta_{1}} \mathrm{e}^{-\mu x} M^{(1)}\left(\omega \mid x, \delta_{1}\right) \mathrm{d} x+\mathrm{e}^{-\mu v_{1}} \tilde{M}^{(1)}\left(\omega \mid \delta_{1}\right) .
$$

Note that

$$
\begin{aligned}
\mu \int_{0}^{\delta_{1}} \mathrm{e}^{-\mu x} \psi_{L}^{*}\left(\omega \mid x, \delta_{1}-x\right) \mathrm{d} x= & \frac{\mu}{\mu+\theta_{2}(\omega)} \zeta_{1}(\omega)\left(1-\mathrm{e}^{-\delta_{1}\left(\mu+\theta_{2}(\omega)\right)}\right) \\
& -\frac{\mu}{\mu+\theta_{1}(\omega)} \zeta_{2}(\omega)\left(1-\mathrm{e}^{-\delta_{1}\left(\mu+\theta_{1}(\omega)\right)}\right) .
\end{aligned}
$$

Also,

$$
\begin{aligned}
\mu \int_{0}^{\delta_{1}} & \mathrm{e}^{-\mu x} \psi_{U}^{*}\left(\omega \mid x, \delta_{1}-x\right) \mathrm{d} x \\
= & \frac{1}{\left(\mu+\theta_{2}(\omega)\right) \mathrm{e}^{-\delta_{1} \theta_{1}(\omega)}-\left(\mu+\theta_{1}(\omega)\right) \mathrm{e}^{-\delta_{1} \theta_{2}(\omega)}} \\
& \times\left[\frac{\mu}{\mu+\theta_{1}(\omega)}\left(1-\mathrm{e}^{-\delta_{1}\left(\mu+\theta_{1}(\omega)\right)}\right)-\frac{\mu}{\mu+\theta_{2}(\omega)}\left(1-\mathrm{e}^{-\delta_{1}\left(\mu+\theta_{2}(\omega)\right)}\right)\right] .
\end{aligned}
$$

\subsection{The busy period LST when $K=2$}

If $K=2$, there are $K+1=3$ regions, $\mathcal{V}_{1}, \mathcal{V}_{2}$, and $\mathcal{V}_{3}$. The instant $V(t)$ enters $\left(\mathcal{V}_{2} \cup \mathcal{V}_{3}\right)$ the process changes the intensity of arrival to $\lambda_{2}=\lambda Q_{1}$ or $\lambda_{3}=0$. The time until the first return to $\mathcal{V}_{1}$ will be called $B^{(2)}$. The LST of $B^{(2)}$ is obtained using (5.7), replacing $\delta_{1}$ by $\delta_{2}=v_{2}-v_{1}, \lambda$ by $\lambda_{2}$, and $v_{1}=\delta_{1}$ by $\delta_{2}$. We denote this LST by $M_{2}^{(1)}\left(\omega \mid \delta_{2}\right)$.

Let $X_{1}$ denote the service requirement of the first customer. Consider first the case where $0<X_{1}<v_{1}$. In this case either $V(t)$ hits 0 before crossing $v_{1}$ or crosses $v_{1}$ first. If $V(t)$ crosses $v_{1}$ before hitting 0 , the 'initial phase' is the time interval from 0 till the first entry back to $\mathcal{V}_{1}$. Renewal cycles are between consecutive re-entrances to $\mathcal{V}_{1}$. Thus, the conditional LST, 
given $X_{1}$, for $X_{1}<v_{1}$, is

$$
\begin{aligned}
M^{(2)}\left(\omega \mid X_{1}, \delta_{1}\right)= & \psi_{L}^{*}\left(\omega \mid X_{1}, \delta_{1}-X_{1}\right) \\
+ & \psi_{U}^{*}\left(\omega \mid X_{1}, \delta_{1}-X_{1}\right) M_{2}^{(1)}\left(\omega \mid \delta_{2}\right) \\
& \quad \times \psi_{L}^{*}\left(\omega \mid \delta_{1}, 0\right)\left[1-\psi_{U}^{*}\left(0 \mid \delta_{1}, 0\right) M_{2}^{(1)}\left(\omega \mid \delta_{2}\right)\right]^{-1}
\end{aligned}
$$

On the other hand, the LST given that $\left\{X_{1} \geq v_{1}\right\}$ is

$$
\tilde{M}^{(2)}\left(\omega \mid \delta_{1}\right)=M_{2}^{(1)}\left(\omega \mid \delta_{2}\right) \psi_{L}^{*}\left(\omega, \delta_{1}, 0\right)\left[1-\psi_{U}^{*}\left(\omega \mid \delta_{1}, 0\right) M_{2}^{(1)}\left(\omega \mid \delta_{2}\right)\right]^{-1} .
$$

Finally, the LST of $B$ for $K=2$ is

$$
M^{(2)}\left(\omega \mid \delta_{1}\right)=\mu \int_{0}^{\delta_{1}} \mathrm{e}^{-\mu x} M^{(2)}\left(\omega \mid x, \delta_{1}\right) \mathrm{d} x+\mathrm{e}^{-\mu \delta_{1}} \tilde{M}^{(2)}\left(\omega \mid \delta_{1}\right) .
$$

\subsection{The busy period LST for general $K$}

We start by computing the LST for the $K$ th region $\mathcal{V}_{K}$, according to formulae (5.5)-(5.9), in which we substitute $\lambda_{K}=\lambda Q_{K-1}$ and $\delta_{K}=v_{K}-v_{K-1}$. We denote this LST as $M_{K}^{(1)}\left(\omega \mid \delta_{K}, \lambda_{K}\right)$. We then proceed to determine the LST for $\mathcal{V}_{K-1}$, namely $M_{K-1}^{(2)}\left(\omega \mid \delta_{K-1}\right.$, $\lambda_{K-1}$ ), according to (5.10)-(5.12). Recursively, for $j=2, \ldots, K$, we compute, for $X<$ $\delta_{K+1-j}$,

$$
\begin{aligned}
& M_{(K+1-j)}^{(j)}\left(\omega \mid X, \delta_{K+1-j}, \lambda_{K+1-j}\right) \\
& =\psi_{L}^{*}\left(\omega \mid X, \delta_{K+1-j}-X, \lambda_{K+1-j}\right) \\
& +\psi_{U}^{*}\left(\omega \mid X, \delta_{K+1-j}-X, \lambda_{K+1-j}\right) M_{K+2-j}^{(j-1)}\left(\omega \mid \delta_{K+2-j}, \lambda_{K+2-j}\right) \\
& \quad \times \psi_{L}^{*}\left(\omega \mid \delta_{K+1-j}, 0, \lambda_{K+1-j}\right) \\
& \quad \times\left[1-\psi_{U}^{*}\left(\omega \mid \delta_{K+1-j}, 0, \lambda_{K+1-j}\right) M_{K+2-j}^{(j-1)}\left(\omega \mid \delta_{K+2-j}, \lambda_{K+2-j}\right)\right]^{-1}
\end{aligned}
$$

and, for $X \geq \delta_{K+1-j}$, we determine

$$
\begin{aligned}
\tilde{M}^{(j)}(\omega \mid & \left.\delta_{K+1-j}, \lambda_{K+1-j}\right) \\
= & M_{K+2-j}^{(j-1)}\left(\omega \mid \delta_{K+2-j}, \lambda_{K+2-j}\right) \psi_{L}^{*}\left(\omega \mid \delta_{K+1-j}, 0, \lambda_{K+1-j}\right) \\
& \times\left[1-\psi_{U}^{*}\left(\omega \mid \delta_{K+1-j}, 0, \lambda_{K+1-j}\right) M_{K+2-j}^{(j-1)}\left(\omega \mid \delta_{K+2-j}, \lambda_{K+2-j}\right)\right]^{-1}
\end{aligned}
$$

and

$$
\begin{aligned}
& M_{K+1-j}^{(j)}\left(\omega \mid \delta_{K+1-j}, \lambda_{K+1-j}\right) \\
& =\mu \int_{0}^{\delta_{K+1-j}} \mathrm{e}^{-\mu x} M_{K+1-j}^{(j)}\left(\omega \mid x, \delta_{K+1-j}-x, \lambda_{K+1-j}\right) \mathrm{d} x \\
& \quad+\mathrm{e}^{-\mu \delta_{K+1-j}} \tilde{M}^{(j)}\left(\omega \mid \delta_{K+1-j}, \lambda_{K+1-j}\right) .
\end{aligned}
$$

In the present section we formulated the variables $\lambda_{K+1-j}, j=1, \ldots, K$, in LST functional form, to emphasize the dependence on the different intensities $\lambda_{l}, l=1, \ldots, K$. 


\section{The busy period LST for an M/G/1 queue with discrete patience}

In this section we no longer assume that service times are exponentially distributed. In determining the busy period LST, we follow the same approach as in the previous section. In Subsection 6.1 we again consider the stopping variables introduced in (5.1) and (5.2). We now also need an expression for the joint distribution of $T_{U}\left(\beta_{2}\right)$ and the overshoot $Y_{2}-\left(\beta_{2}+T_{U}\left(\beta_{2}\right)\right)$, which is no longer exponentially distributed. We subsequently consider $K=1$ (deterministic patience), $K=2$, and general $K$, in Subsections 6.2, 6.3, and 6.4, respectively.

\subsection{Auxiliary results}

The arrival of customers at the queue follows a homogeneous Poisson process $\{N(t), t \geq 0\}$ with intensity $\lambda$. The required service times of customers are i.i.d. random variables $X_{1}, X_{2}, \ldots$ having distribution $F$, with density $f$. Let $Y_{t}=\sum_{n=0}^{N(t)} X_{n}$, where $X_{0} \equiv 0$. The density of $Y_{t}$ on $(0, \infty)$ is $h_{\lambda}(y ; t)=\sum_{n=1}^{\infty} p(n ; \lambda t) f^{(n)}(y)$, where $p(n ; \lambda t)$ is the probability density function of Poisson $(\lambda t)$ and $f^{(n)}(y)$ is the $n$ th-fold convolution of $f$ at $y$, i.e. $f^{(n)}(y)=$ $\int_{0}^{y} f^{(n-1)}(y-x) f(x) \mathrm{d} x, n \geq 1$, where $f^{(0)}(y) \equiv 1$. Let $H_{\lambda}(y ; t)$ denote the CDF of $Y_{t}$, i.e. $H_{\lambda}(y, t)=\sum_{n=0}^{\infty} p(n ; \lambda t) F^{(n)}(y)$. Note that $H_{\lambda}(y, t)$ has an atom at $y=0, H_{\lambda}(0 ; t)=$ $\mathrm{e}^{-\lambda t}$, and $H_{\lambda}(y, t)$ is absolutely continuous on $(0, \infty)$. For nonnegative constants $0 \leq \beta_{1}$, $\beta_{2}<\infty$, define the stopping variables

$$
T_{L}\left(\beta_{1}\right)=\inf \left\{t \geq 0: Y_{t}=-\beta_{1}+t\right\}
$$

and

$$
T_{U}\left(\beta_{2}\right)=\inf \left\{t \geq 0: Y_{t} \geq \beta_{2}+t\right\} .
$$

Moreover, let $T\left(\beta_{1}, \beta_{2}\right)=\min \left\{T_{L}\left(\beta_{1}\right), T_{U}\left(\beta_{2}\right)\right\}$. We need explicit equations for the LSTs

$$
\psi_{L}^{*}\left(\omega ; \beta_{1}, \beta_{2}, \lambda\right)=\mathrm{E}_{\lambda}\left(\mathrm{e}^{-\omega T_{L}\left(\beta_{1}\right)} \mathbf{1}_{\left\{T_{L}\left(\beta_{1}\right)<T_{U}\left(\beta_{2}\right)\right\}}\right)
$$

and

$$
\psi_{U}^{*}\left(\omega ; \beta_{1}, \beta_{2}, \lambda\right)=\mathrm{E}_{\lambda}\left(\mathrm{e}^{-\omega T_{U}\left(\beta_{2}\right)} \mathbf{1}_{\left\{T_{L}\left(\beta_{1}\right)>T_{U}\left(\beta_{2}\right)\right\}}\right) .
$$

Moreover, we need a formula for the joint distribution of $T_{U}\left(\beta_{2}\right)$ and the overshoot $R=$ $Y_{T_{U}\left(\beta_{2}\right)}-\left(\beta_{2}+T_{U}\left(\beta_{2}\right)\right)$. These equations are given below.

Let

$$
g_{\beta_{2}}(y ; t, \lambda)=\frac{\mathrm{d}}{\mathrm{d} y} \mathrm{P}_{\lambda}\left\{Y_{t} \leq y, T_{U}\left(\beta_{2}\right)>t\right\}
$$

and

$$
g_{\lambda}\left(y ; t, \beta_{1}, \beta_{2}\right)=\frac{\mathrm{d}}{\mathrm{d} y} \mathrm{P}_{\lambda}\left\{Y_{t} \leq y, T\left(\beta_{1}, \beta_{2}\right)>t\right\} .
$$

As proven by Stadje and Zacks [11],

$$
g_{0}(y ; t, \lambda)=\frac{(t-y)^{+}}{t} h_{\lambda}(y ; t), \quad t>0 .
$$

Moreover, for $\beta_{2}>0$ and $0<y<t+\beta_{2}$,

$$
\begin{aligned}
g_{\beta_{2}}(y ; t, \lambda)=h_{\lambda}(y ; t)-\mathbf{1}_{\left(\beta_{2}, \beta_{2}+t\right)}(y) & {\left[\mathrm{e}^{-\lambda\left(t+\beta_{2}-y\right)} h_{\lambda}\left(y ; y-\beta_{2}\right)\right.} \\
& \left.+\int_{\beta_{2}}^{y} h_{\lambda}\left(u ; u-\beta_{2}\right) g_{0}\left(y-u ; t+\beta_{2}-u, \lambda\right) \mathrm{d} u\right] .
\end{aligned}
$$


The function $g_{\lambda}\left(y ; t, \beta_{1}, \beta_{2}\right)$ can be written in terms of $g_{\beta}(y ; t, \lambda)$. Let $\delta=\beta_{1}+\beta_{2}$. Then, for $\left(t-\beta_{1}\right)^{+}<y<t+\beta_{2}$,

$$
\begin{aligned}
g_{\lambda}\left(y ; t, \beta_{1}, \beta_{2}\right)= & g_{\beta_{2}}(y ; t, \lambda) \\
& \quad-\mathbf{1}_{\left(\beta_{1}, \infty\right)}(t)\left[\mathrm{e}^{-\lambda \beta_{1}} g_{\delta}\left(y ; t-\beta_{1}, \lambda\right)\right. \\
& \left.\quad+\beta_{1} \int_{\beta_{1}}^{y} \frac{1}{s} g_{\beta_{2}}\left(s-\beta_{1} ; s, \lambda\right) g_{\delta}\left(y-s+\beta_{1} ; t-s, \lambda\right) \mathrm{d} s\right] .
\end{aligned}
$$

The joint density of $\left(T_{U}\left(\beta_{2}\right), R\right)$ is then

$$
\begin{aligned}
p_{\lambda}\left(t, r ; \beta_{1}, \beta_{2}\right) & \\
= & \mathbf{1}_{\left\{t \leq \beta_{1}\right\}}\left[\lambda \mathrm{e}^{-\lambda t} f\left(t+\beta_{2}+r\right)+\lambda \int_{0}^{t+\beta_{2}} g_{\beta_{2}}(y ; t, \lambda) f\left(t+\beta_{2}+r-y\right) \mathrm{d} y\right] \\
& +\mathbf{1}_{\left\{t>\beta_{1}\right\}} \lambda \int_{t-\beta_{1}}^{t+\beta_{2}} g_{\lambda}\left(y ; t, \beta_{1}, \beta_{2}\right) f\left(t+\beta_{2}+r-y\right) \mathrm{d} y .
\end{aligned}
$$

In addition, as in [4],

$$
\psi_{L}^{*}\left(\omega ; \beta_{1}, \beta_{2}, \lambda\right)=\mathrm{e}^{-(\lambda+\omega) \beta_{1}}+\int_{\beta_{1}}^{\infty} \mathrm{e}^{-\omega t} \frac{t-\beta_{1}}{t} g_{\beta_{2}}\left(t-\beta_{1} ; t, \lambda\right) \mathrm{d} t .
$$

Similarly,

$$
\begin{aligned}
\psi_{U}^{*}\left(\omega ; \beta_{1}, \beta_{2}, \lambda\right)= & \lambda \int_{0}^{\beta_{1}} \mathrm{e}^{-(\lambda+\omega) t} \bar{F}\left(t+\beta_{2}\right) \mathrm{d} t \\
& +\lambda \int_{0}^{\beta_{1}} \mathrm{e}^{-\omega t} \int_{0}^{t+\beta_{2}} g_{\beta_{2}}(y ; t, \lambda) \bar{F}\left(t+\beta_{2}-y\right) \mathrm{d} y \mathrm{~d} t \\
& +\lambda \int_{\beta_{1}}^{\infty} \mathrm{e}^{-\omega t} \int_{t-\beta_{1}}^{t+\beta_{2}} g_{\lambda}\left(y ; t, \beta_{1}, \beta_{2}\right) \bar{F}\left(t+\beta_{2}-y\right) \mathrm{d} y \mathrm{~d} t .
\end{aligned}
$$

\subsection{The busy period LST when $K=1$}

The case in which $K=1$ corresponds to an M/G/1 queue with deterministic $\left(v_{1}\right)$ patience; see also Model II of [8] for this case. We have

$$
T_{U}^{(1)}=T_{U}\left(v_{1}-X_{1}\right), \quad T_{U}^{(2)}=T_{U}^{(1)}+R^{(1)}+T_{U}(0) .
$$

In the case $T_{U}^{(1)}<T_{L}^{(0)}\left(X_{1}\right)$ and $0<X_{1}<v_{1}$ we have an initial phase $C_{I}$ consisting of $T_{U}^{(1)}$ and $R_{1}$. The point $T_{U}^{(1)}+R_{1}$ is a regeneration point, where a new phase starts. We denote it as $C_{R}$. If $T_{L}^{(2)}\left(v_{1}\right)<T_{U}^{(2)}(0)$, the busy period ends; otherwise the phase $C_{R}$ consists of $T_{U}^{(2)}(0)+R_{2}$, etc.

We define, for $0<X<v_{1}$,

$$
\psi_{U I}^{*}\left(\omega ; X, v_{1}-X, \lambda_{1}\right)=\int_{0}^{\infty} \mathrm{e}^{-\omega t} \int_{0}^{\infty} \mathrm{e}^{-\omega r} p_{\lambda_{1}}\left(t, r ; X, v_{1}-X\right) \mathrm{d} r \mathrm{~d} t .
$$

Similarly, let

$$
\psi_{U R}^{*}\left(\omega ; v_{1}, \lambda_{1}\right)=\int_{0}^{\infty} \mathrm{e}^{-\omega t} \int_{0}^{\infty} \mathrm{e}^{-\omega r} p_{\lambda_{1}}\left(t, r ; v_{1}, 0\right) \mathrm{d} r \mathrm{~d} t
$$


The conditional LST of $B$, when $0<X_{1}<v_{1}$, is then

$$
\begin{aligned}
M_{I}^{(1)}\left(\omega ; X_{1}, v_{1}-X_{1}, \lambda_{1}\right)= & \psi_{L}^{*}\left(\omega ; X_{1}, v_{1}-X_{1}, \lambda_{1}\right) \\
& +\psi_{U I}^{*}\left(\omega ; X_{1}, v_{1}-X_{1}, \lambda_{1}\right) \frac{\psi_{L}^{*}\left(\omega ; v_{1}, 0, \lambda_{1}\right)}{\psi_{U R}^{*}\left(\omega ; v_{1}, \lambda_{1}\right)} .
\end{aligned}
$$

In the case $v_{1} \leq X_{1}<\infty$, the conditional LST of $B$ is

$$
M_{I I}^{(1)}\left(\omega ; X_{1}, \lambda_{1}\right)=\mathrm{e}^{-\omega\left(X_{1}-v_{1}\right)} \frac{\psi_{L}^{*}\left(\omega ; v_{1}, 0, \lambda_{1}\right)}{\psi_{U R}^{*}\left(\omega ; v_{1}, \lambda_{1}\right)} .
$$

Finally, the LST of $B$ is

$$
\begin{aligned}
M^{(1)}\left(\omega ; v_{1}, \lambda_{1}\right)= & \int_{0}^{v_{1}} f(x) \psi_{L}^{*}\left(\omega ; x, v_{1}-x, \lambda_{1}\right) \mathrm{d} x \\
& +\frac{\psi_{L}^{*}\left(\omega ; v_{1}, 0, \lambda_{1}\right)}{\psi_{U R}^{*}\left(\omega ; v_{1}, \lambda_{1}\right)}\left(\int_{0}^{v_{1}} f(x) \psi_{U I}^{*}\left(\omega ; x, v_{1}-x, \lambda_{1}\right) \mathrm{d} x\right. \\
& \left.+\mathrm{e}^{\omega v_{1}} \int_{v_{1}}^{\infty} f(x) \mathrm{e}^{-\omega x} \mathrm{~d} x\right) .
\end{aligned}
$$

\subsection{The busy period LST when $K=2$}

For $0<X_{1}<v_{1}$, the conditional LST is, with $\delta_{2}=v_{2}-v_{1}$,

$$
\begin{aligned}
& M_{I}^{(2)}\left(\omega ; X_{1}, v_{1}-X_{1}, \lambda_{1}\right) \\
& =\psi_{L}^{*}\left(\omega ; X_{1}, v_{1}-X_{1}, \lambda_{1}\right) \\
& +\left[\int_{0}^{\infty} \mathrm{e}^{-\omega t} \int_{0}^{\delta_{2}} M_{I}^{(1)}\left(\omega ; r, \delta_{2}-r, \lambda_{2}\right) p_{\lambda_{1}}\left(t, r ; X_{1}, v_{1}-X_{1}\right) \mathrm{d} r \mathrm{~d} t\right. \\
& \left.\quad+\int_{0}^{\infty} \mathrm{e}^{-\omega t} \int_{\delta_{2}}^{\infty} M_{I I}^{(1)}\left(\omega ; r, \lambda_{2}\right) p_{\lambda_{1}}\left(t, r ; X_{1}, v_{1}-X_{1}, \lambda_{1}\right) \mathrm{d} r \mathrm{~d} t\right] \\
& \quad+\left[\int_{0}^{\infty} \mathrm{e}^{-\omega t} \int_{0}^{\delta_{2}} M_{I}^{(1)}\left(\omega ; r, \delta_{2}-r, \lambda_{2}\right) p_{\lambda_{1}}\left(t, r ; v_{1}, 0\right) \mathrm{d} r \mathrm{~d} t\right. \\
& \left.\quad+\int_{0}^{\infty} \mathrm{e}^{-\omega t} \int_{\delta_{2}}^{\infty} M_{I I}^{(1)}\left(\omega ; r, \lambda_{2}\right) p_{\lambda_{1}}\left(t, r ; v_{1}, 0\right) \mathrm{d} r \mathrm{~d} t\right]^{-1} \psi_{L}^{*}\left(\omega ; v_{1}, 0, \lambda_{1}\right) .
\end{aligned}
$$

For $X_{1} \geq v_{1}$, the conditional LST is

$$
\begin{aligned}
& M_{I I}^{(2)}\left(\omega ; X_{1}, \lambda_{1}\right) \\
& =\left[\mathbf{1}_{\left\{v_{1}<X_{1}<v_{2}\right\}} M_{I}^{(1)}\left(\omega ; X_{1}-v_{1}, v_{2}-X_{1}, \lambda_{2}\right)\right. \\
& \left.\quad+\mathbf{1}_{\left\{X_{1} \geq v_{2}\right\}} M_{I I}^{(1)}\left(\omega ; X_{1}-v_{2}, \lambda_{2}\right)\right] \\
& \quad \times\left[\int_{0}^{\infty} \mathrm{e}^{-\omega t} \int_{0}^{\delta_{2}} M_{I}^{(1)}\left(\omega ; r, \delta_{2}-r, \lambda_{2}\right) p_{\lambda_{1}}\left(t, r ; v_{1}, 0\right) \mathrm{d} r \mathrm{~d} t\right. \\
& \left.\quad+\int_{0}^{\infty} \mathrm{e}^{-\omega t} \int_{\delta_{2}}^{\infty} M_{I I}^{(1)}\left(\omega, r, \lambda_{2}\right) p_{\lambda_{1}}\left(t, r ; v_{1}, 0\right) \mathrm{d} r \mathrm{~d} t\right]^{-1} \psi_{L}^{*}\left(\omega ; v_{1}, 0, \lambda_{1}\right) .
\end{aligned}
$$

Finally, the LST of the busy period length, when $K=2$, is

$$
M^{(2)}\left(\omega ; v_{1}, \lambda_{1}\right)=\int_{0}^{v_{1}} f(x) M_{I}^{(2)}\left(\omega ; x, v_{1}-x, \lambda_{1}\right) \mathrm{d} x+\int_{v_{1}}^{\infty} M_{I I}^{(2)}\left(\omega ; x, \lambda_{1}\right) f(x) \mathrm{d} x .
$$




\subsection{The busy period LST for general $K$}

In the general case we have $\delta_{j}=v_{j}-v_{j-1}, j=1, \ldots, K\left(v_{0} \equiv 0\right)$, and $\lambda_{j}=\lambda Q_{j-1}, j=$ $1, \ldots, K$. We first compute $M_{I}^{(1)}\left(\omega ; x, \delta_{k}-x, \lambda_{K}\right)$ and $M_{I I}^{(1)}\left(\omega ; x, \lambda_{K}\right)$ according to (6.1) and (6.2). Afterwards, for each $j=1, \ldots, K$, we compute recursively the functions

$$
\begin{aligned}
M_{U I}^{(j)}\left(\omega ; x, \delta_{j}, \lambda_{j}\right)= & \int_{0}^{\infty} \mathrm{e}^{-\omega t} \int_{0}^{\delta_{j+1}} M_{I}^{(j-1)}\left(\omega ; r, \delta_{j+1}-r, \lambda_{j+1}\right) p_{\lambda_{j}}\left(t, r ; x, \delta_{j}-x\right) \mathrm{d} r \mathrm{~d} t \\
& +\int_{0}^{\infty} \mathrm{e}^{-\omega t} \int_{\delta_{j+1}}^{\infty} M_{I I}^{(j-1)}\left(\omega ; r, \lambda_{j+1}\right) p_{\lambda_{j}}\left(t, r ; x, \delta_{j}-x\right) \mathrm{d} r \mathrm{~d} t, \\
D^{(j)}\left(\omega ; \lambda_{j}\right)= & \int_{0}^{\infty} \mathrm{e}^{-\omega t} \int_{0}^{\delta_{j+1}} M_{I}^{(j-1)}\left(\omega ; r, \delta_{j+1}-r, \lambda_{j+1}\right) p_{\lambda_{j}}\left(t, r ; \delta_{j}, 0\right) \mathrm{d} r \mathrm{~d} t \\
& +\int_{0}^{\infty} \mathrm{e}^{-\omega t} \int_{\delta_{j+1}}^{\infty} M_{I I}^{(j-1)}\left(\omega ; r, \lambda_{j+1}\right) p_{\lambda_{j}}\left(t, r ; \delta_{j}, 0\right) \mathrm{d} r \mathrm{~d} t,
\end{aligned}
$$

and

$$
\begin{gathered}
M_{I}^{(j)}\left(\omega ; x, \delta_{j}-x, \lambda_{j}\right)=\psi_{L}^{*}\left(\omega ; x, \delta_{j}-x, \lambda_{j}\right)+\frac{M_{U I}^{(j)}\left(\omega ; x, \delta_{j}, \lambda_{j}\right)}{D^{(j)}\left(\omega ; \lambda_{j}\right)} \psi_{L}^{*}\left(\omega ; \delta_{j}, 0, \lambda_{2}\right), \\
M_{I I}^{(j)}\left(\omega ; x, \lambda_{j}\right)=\left[\mathbf{1}_{\left\{v_{j}<x<v_{j+1}\right\}} M_{I}^{(k-1)}\left(\omega ; x-v_{j}, v_{j+1}-x, \lambda_{j+1}\right)\right. \\
\left.+\mathbf{1}_{\left\{x \geq v_{j+1}\right\}} M_{I I}^{(k-1)}\left(\omega ; x-v_{j+1}, \lambda_{j+1}\right)\right] \frac{\psi_{L}^{*}\left(\omega ; \delta_{j}, 0, \lambda_{j}\right)}{D^{(j)}\left(\omega ; \lambda_{j}\right)} .
\end{gathered}
$$

Finally, the LST of $B$ is

$$
M^{(K)}(\omega ; \lambda)=\int_{0}^{v_{1}} f(x) M_{I}^{(K)}\left(\omega ; x, v_{1}-x, \lambda_{1}\right) \mathrm{d} x+\int_{v_{1}}^{\infty} f(x) M_{I I}^{(K)}\left(\omega ; x, \lambda_{1}\right) \mathrm{d} x .
$$

Note that $\lambda_{1} \equiv \lambda$.

\section{Acknowledgements}

We are grateful to the anonymous referee, whose comments helped improve the presentation of the paper. The research of O. J. Boxma was done within the framework of the BRICKS project and the European Network of Excellence Euro-FGI. W. Stadje was supported by the Deutsche Forschungsgemeinschaft.

\section{References}

[1] Baccelli, F. And Hébuterne, G. (1981). On queues with impatient customers. In Performance '81 (Amsterdam, 1981), ed. F. J. Kylstra, North Holland, Amsterdam, pp. 159-179.

[2] Baccelli, F., Boyer, P. and Hébuterne, G. (1984). Single-server queues with impatient customers. Adv. Appl. Prob. 16, 887-905.

[3] Barrer, D. Y. (1957). Queuing with impatient customers and ordered service. Operat. Res. 5, 650-656.

[4] Borovkov, K. ANd BurQ, Z. (2001). Kendall's identity for the first crossing time revisited. Electron. Commun. Prob. 6, 91-94.

[5] FINCH, P. D. (1960). Deterministic customer impatience in the queueing system GI / M/1. Biometrika 47, 45-52.

[6] Kaspi, H. and Perry, D. (1983). Inventory systems of perishable commodities. Adv. Appl. Prob. 15, 674-685.

[7] Perry, D. And Asmussen, S. (1995). Rejection rules in the M/G/1 queue. Queueing Systems 19, $105-130$.

[8] Perry, D., Stadje, W. And Zacks, S. (2000). Busy period analysis for $M / G / 1$ and $G / M / 1$ type queues with restricted accessibility. Operat. Res. Lett. 27, 163-174. 
[9] Perry, D., Stadje, W. ANd Zacks, S. (2001). The $M / G / 1$ queue with finite workload capacity. Queueing Systems 39, 7-22.

[10] Ross, S. M. (1996). Stochastic Processes, 2nd edn. John Wiley, New York.

[11] Stadje, W. and Zacks, S. (2003). Upper first-exit times of compound Poisson processes revisited. Prob. Eng. Inf. Sci. 17, 459-465.

[12] Stanford, R. E. (1979). Reneging phenomenon in single channel queues. Math. Operat. Res. 4, $162-178$.

[13] Stanford, R. E. (1990). On queues with impatience. Adv. Appl. Prob. 22, 768-769.

[14] Subba Rao, S. (1967). Queueing models with balking and reneging. Ann. Inst. Statist. Math. 19, 55-71.

[15] Subba RaO, S. (1967/1968). Queuing with balking and reneging in MG1 systems. Metrika 12, $173-188$.

[16] Widder, D. V. (1946). The Laplace Transform. Princeton University Press. 